\title{
OPEN Cochlear shape distinguishes southern African early hominin taxa with unique auditory ecologies
}

\author{
J. Braga ${ }^{1,2 \bowtie}$, C. Samir ${ }^{3}$, A. Fradi ${ }^{3}$, Y. Feunteun ${ }^{3}$, K. Jakata², V. A. Zimmer ${ }^{4}$, B. Zipfel ${ }^{2}$, \\ J. F. Thackeray' ${ }^{2}$ M. Macé ${ }^{5}$, B. A. Wood ${ }^{6}$ \& F. E. Grine ${ }^{7,8}$
}

Insights into potential differences among the bony labyrinths of Plio-Pleistocene hominins may inform their evolutionary histories and sensory ecologies. We use four recently-discovered bony labyrinths from the site of Kromdraai to significantly expand the sample for Paranthropus robustus. Diffeomorphometry, which provides detailed information about cochlear shape, reveals sizeindependent differences in cochlear shape between $P$. robustus and Australopithecus africanus that exceed those among modern humans and the African apes. The cochlea of $P$. robustus is distinctive and relatively invariant, whereas cochlear shape in $A$. africanus is more variable, resembles that of early Homo, and shows a degree of morphological polymorphism comparable to that evinced by modern species. The curvature of the $P$. robustus cochlea is uniquely derived and is consistent with enhanced sensitivity to low-frequency sounds. Combined with evidence for selection, our findings suggest that sound perception shaped distinct ecological adaptations among southern African early hominins.

The morphology of the bony labyrinth reflects the shape of its membranous compartments, whose receptors detect sound in the cochlea, and sense head position and motion in the vestibule and semi-circular canals, respectively ${ }^{1-3}$. The developmental stability ${ }^{4}$ and species-specific features ${ }^{5-7}$ of the bony labyrinth, and the relationships between cochlear morphology and auditory perception among mammalian species ${ }^{8-10}$, suggest that cochlear shape has the potential to provide evidence about the sensory ecology of extinct hominin taxa. Previous investigations have not detected significant cochlear differences among Plio-Pleistocene australopiths ${ }^{1,2,11}$. However, the complex shape of the cochlea, comprising a curve of decreasing radius that approximates but does not conform precisely to a logarithmic spiral with different degrees of torsion along its length, does not lend itself to being captured by the linear methods and 3D geometric morphometric (3DGM) approaches that were previously employed.

Here, we used diffeomorphometry ${ }^{12-14}$, which has detected sex-based and size-independent cochlear differences in modern humans ${ }^{15}$, to investigate potential differences in cochlear morphology among fossil hominin taxa from southern Africa. We analyze cochlear shape using a non-linear geometric framework that employs Fréchet means to define the optimal size-independent differences between complex shapes ("Methods"). The Fréchet mean ${ }^{12,13}$ of two points along a curved surface is located at equal geodesic distances (arc lengths) between them, whereas the standard arithmetic mean utilized in 3DGM analyses is located at mid-linear distance, and therefore lies outside the curve. This non-linear geometric framework, unlike 3DGM, allows measurements of local geometric properties ${ }^{16}$, including mapping changes in curvature (bending) and torsion (twisting) at any location along the length of the cochlea, from its base to the apex ("Methods"). We applied this method to microCT images of the cochlea of samples of museum specimens representing modern humans (Homo sapiens, $\mathrm{n}=16$ ), common (Pan troglodytes, $\mathrm{n}=16$ ) and pygmy (Pan paniscus, $\mathrm{n}=16$ ) chimpanzees, gorillas (Gorilla gorilla, $\mathrm{n}=15$ ), and Plio-Pleistocene hominin fossils from southern African cave sites belonging to Australopithecus africanus $(\mathrm{n}=9)$, Paranthropus robustus $(\mathrm{n}=8)$ and undoubted early Homo $(\mathrm{n}=1)$ (Tables S1,2). The Fréchet mean of $P$.

\footnotetext{
${ }^{1}$ Centre d'Anthropobiologie et de Génomique de Toulouse, Université Paul Sabatier Toulouse III, Faculté de Médecine Purpan, 37 allées Jules Guesde, Toulouse, France. ${ }^{2}$ Evolutionary Studies Institute, University of the Witwatersrand, PO WITS, Johannesburg 2050, South Africa. ${ }^{3}$ LIMOS, UMR 6158 CNRS-Université Clermont Auvergne, 63173 Aubière, France. ${ }^{4}$ Faculty of Informatics, Technical University of Munich, Munich, Germany. ${ }^{5}$ Véto 31, 73 Avenue du Général de Gaulle, 47000 Agen, France. ${ }^{6}$ Center for the Advanced Study of Human Paleobiology, George Washington University, Washington, DC 20052, USA. ${ }^{7}$ Department of Anthropology, Stony Brook University, Stony Brook, NY 11794, USA. ${ }^{8}$ Department of Anatomical Sciences, Stony Brook University, Stony Brook, NY 11794, USA. ${ }^{凶}$ email: jose.braga@univ-tlse3.fr
} 


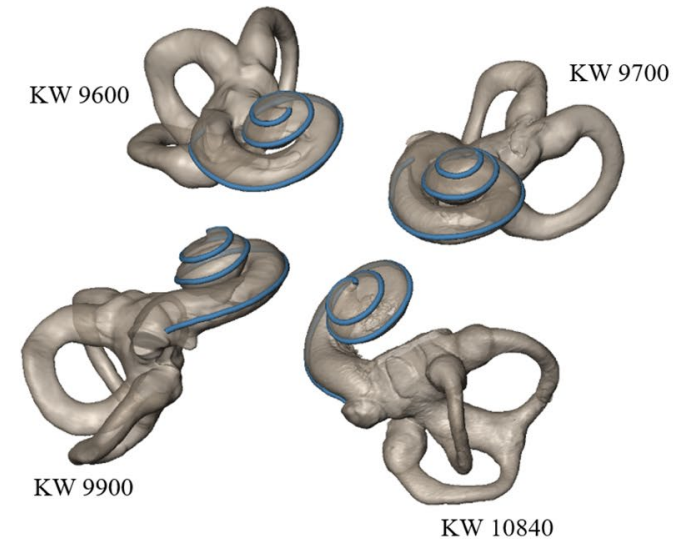

A

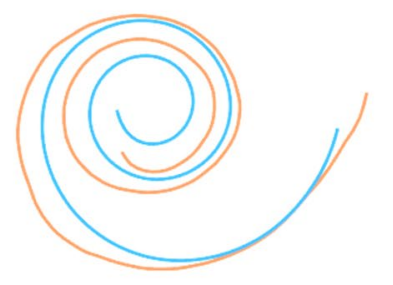

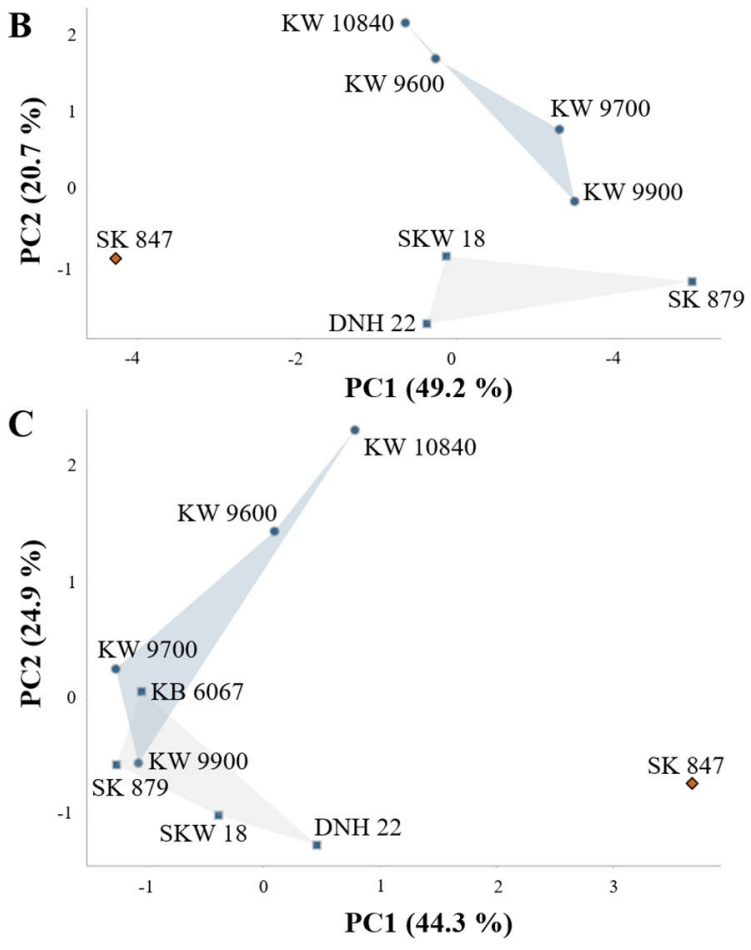

Figure 1. Four new fossil hominins recovered from Kromdraai (South Africa) attributed to Paranthropus robustus, and mean cochlear shape in this species. The new specimens from Kromdraai represent three juvenile (KW 9600, KW 9700 and 10840) and one adult (KW 9990) individuals (blue circles). Blue squares represent other P. robustus specimens from Kromdraai, Swartkrans and Drimolen. Only SK 847 is attributed to early Homo (orange diamond). (A) Fréchet mean computed from our P. robustus sample (blue) aligned with the cochlear curve of the SK 847 early Homo specimen (orange) using exactly the same reference system and orientation. (B,C) Two distinct principal components analyses (PCA, with the variance percentages indicated for PC1 and PC2) using (B) one data set with the two angles and the seven indices listed in Table S3 (the variables that contribute most significantly to PC1 are, with decreasing loadings: TLI, HZL/ANL, ECL/POL, $\mathrm{APA}<\mathrm{LSCm}$ and ECL/ANL); (C) the previous data set adding the incomplete bony labyrinth of KB 6067 (with its damaged anterior semi-circular canal) from Kromdraai ${ }^{27}$ and excluding the HZL/ANL, POL/ANL and ECL/ ANL indices (the variables that contribute most significantly to $\mathrm{PC} 1$ are, with decreasing loadings: $\mathrm{COs}<\mathrm{LSCm}$ and TLI).

robustus cochlear shape is illustrated in Fig. 1. The Fréchet means and curvature and torsion maps for each of the five genera (Australopithecus, Paranthropus, Homo, Pan and Gorilla) in our sample are illustrated in Fig. 2.

While most researchers agree that the australopith remains from Sterkfontein represent a single taxon, A. africanus ${ }^{17,18}$, others have suggested they sample two species of Australopithecus, one of which more closely "approaches" the morphology of Paranthropus ${ }^{19}$, but this latter suggestion has yet to convince most scientists $\mathrm{s}^{17,18,20-22}$. The A. africanus bony labyrinth sample includes nine specimens from Sterkfontein, five of which (StW 252/255/259, StW 498, StW 504/505, StW 573 and StW 578) have been referred by one worker $\left(\right.$ Clarke $\left.^{19}\right)$ to a second species with purported robust australopith affinities. There has been debate over the identification and specific attributions of early Homo fossils from Sterkfontein (specimens StW 53 and StW 151) and Swartkrans (specimen SK 847). While there is consensus that SK 847 belongs to early Homo ${ }^{1,2}$, the attribution of the two specimens from Sterkfontein is somewhat more contentious ${ }^{23-25}$, with some suggesting that they represent $A$. africanus. Here, we considered the StW 53 and StW 151 specimens as taxonomically indeterminate because of the disagreement relating to their assignation.

\section{Results}

Cochlear shape and early hominin taxonomy in southern Africa. The four recently-discovered bony labyrinths from Kromdraai (KW 9600, KW 9700, KW 9900 and KW 10840) included in this study (Fig. 1 and S1) considerably expand our knowledge of bony cochlear shape variation within P. robustus ${ }^{20-22}$. In a principal components analysis (PCA) of bony labyrinthine features, they and the other P. robustus specimens are wellseparated from early Homo on PC1 (Fig. 1 and "Methods"). The features the new specimens from Kromdraai share with other $P$. robustus fossils, and which differentiate them from early Homo, include a smaller transverse labyrinthine index, a less inclined ampular line and a less inclined cochlear basal turn relative to the orientation of the horizontal semi-circular canal, plus larger ECL/POL and ECL/ANL indices ("Methods" and Table S3).

We combined the juvenile and adult specimens in our samples because among modern humans, cochlear shape (assessed with diffeomorphometry) is age independent from birth ${ }^{15}$. In the PCA of bony labyrinthine features (with the first two PC scores), there is no difference between the three new juvenile (KW 9600, KW 9700, 


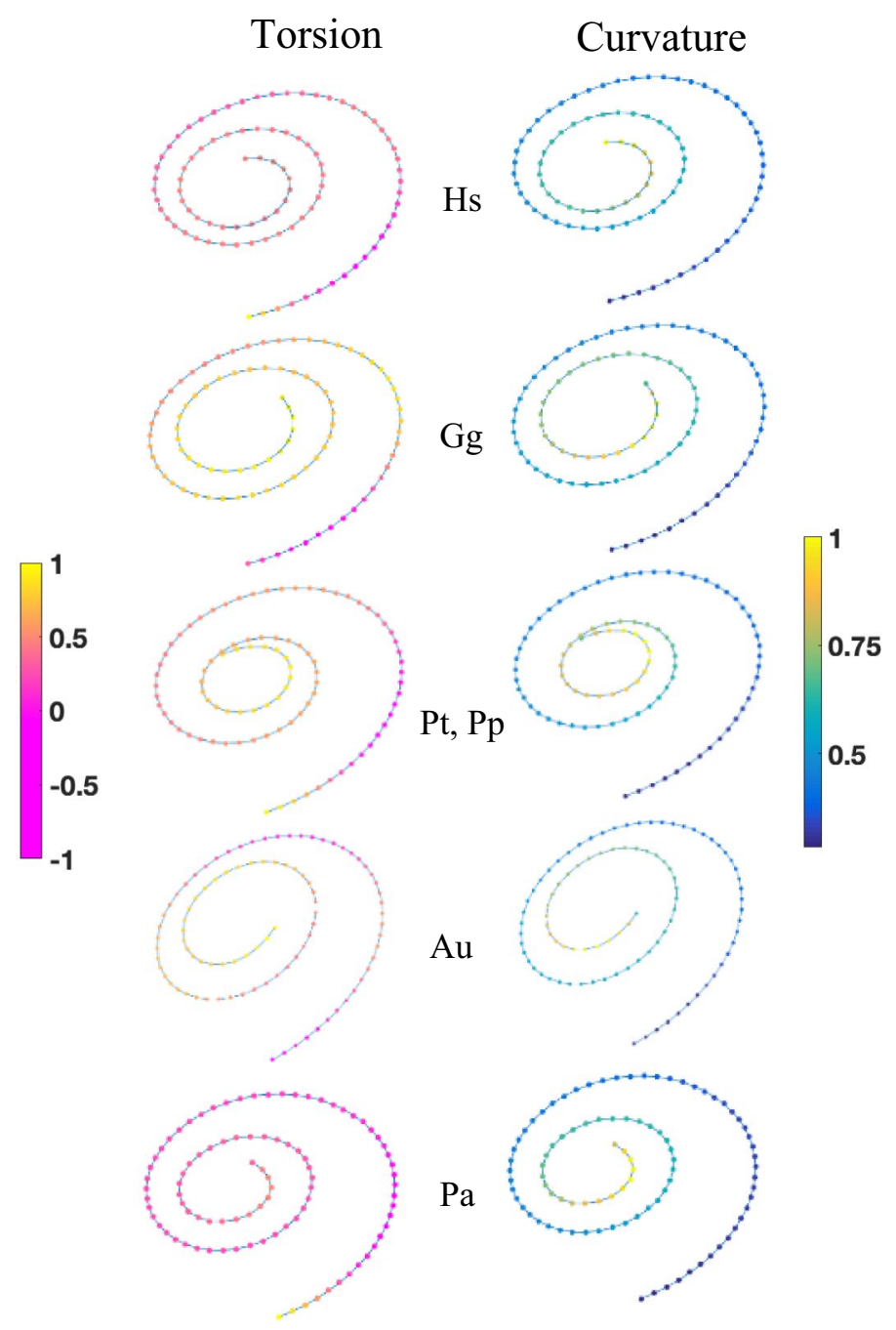

Figure 2. Curvature and torsion of mean cochlear shapes. Mapping curvature (blue to yellow) and torsion (purple to yellow) changes along the Fréchet means from basal to apical locations. Hs Homo sapiens, Gg Gorilla gorilla, Pp Pan paniscus, Pt Pan troglodytes, Au Australopithecus, Pa Paranthropus.

KW 10840) and the new adult (KW 9900) specimen from Kromdraai (Fig. 1), and nor are there any consistent differences between juveniles and adults in the combined P. robustus sample. There is also no consistent difference in cochlear shapes between juveniles and adults in the A. africanus sample (Fig. 3).

A PCA on the tangent space (TPCA $)^{13,15}$, which summarizes most of the variation in cochlear shape (Fig. 3 ), yields better discrimination than 3DGM (Fig. S2). On PC 1, which captures c.83\% of the variance, all seven $P$. robustus specimens form a tight cluster well away from any other group, although they overlap with chimpanzees on PC 2, which captures c.10\% of the variance. Australopithecus specimens, which show a greater range of variation on PC 1, occupy an intermediate position between $P$. robustus and the extant taxa, particularly $H$. sapiens and Gorilla.

Classification and clustering (evaluated by the balanced accuracy and the V-measure) using the first three PCs (97\% of the total variance) of the TPCA ("Methods") results in three clusters comprising, respectively, (i) extant species, (ii) Australopithecus and early Homo, and (iii) P. robustus (Fig. 4). In one cluster, the P. robustus specimens show an exceptionally low level of diversity. In the second cluster, the five specimens from Sterkfontein that have been suggested to represent a second species ${ }^{19}$ are not separated from the four other $A$. africanus fossils (i.e., they belong to the same cluster as all other $A$. africanus fossils, and show close similarities to the StW 329 A. africanus specimen) (Fig. 4), and they are less P. robustus-like than other specimens (Sts 5, Sts 19 and StW 98) from the site (Fig. 3). When compared to the variability in cochlear morphology within modern humans, chimpanzees or gorillas, the Australopithecus sample from Sterkfontein does not show more variation (Figs. 3 and 4). This result is consistent with the hypothesis that the cochlear variability sampled within the Australopithecus sample from Sterkfontein can be accommodated within a single species ${ }^{18}$. Here, too, there is no evidence of an age effect, with the younger StW 98, and the older StW 329 and StW 255 fossils grouping together within the Australopithecus sample. While it could be argued that our results confirm the australopith 


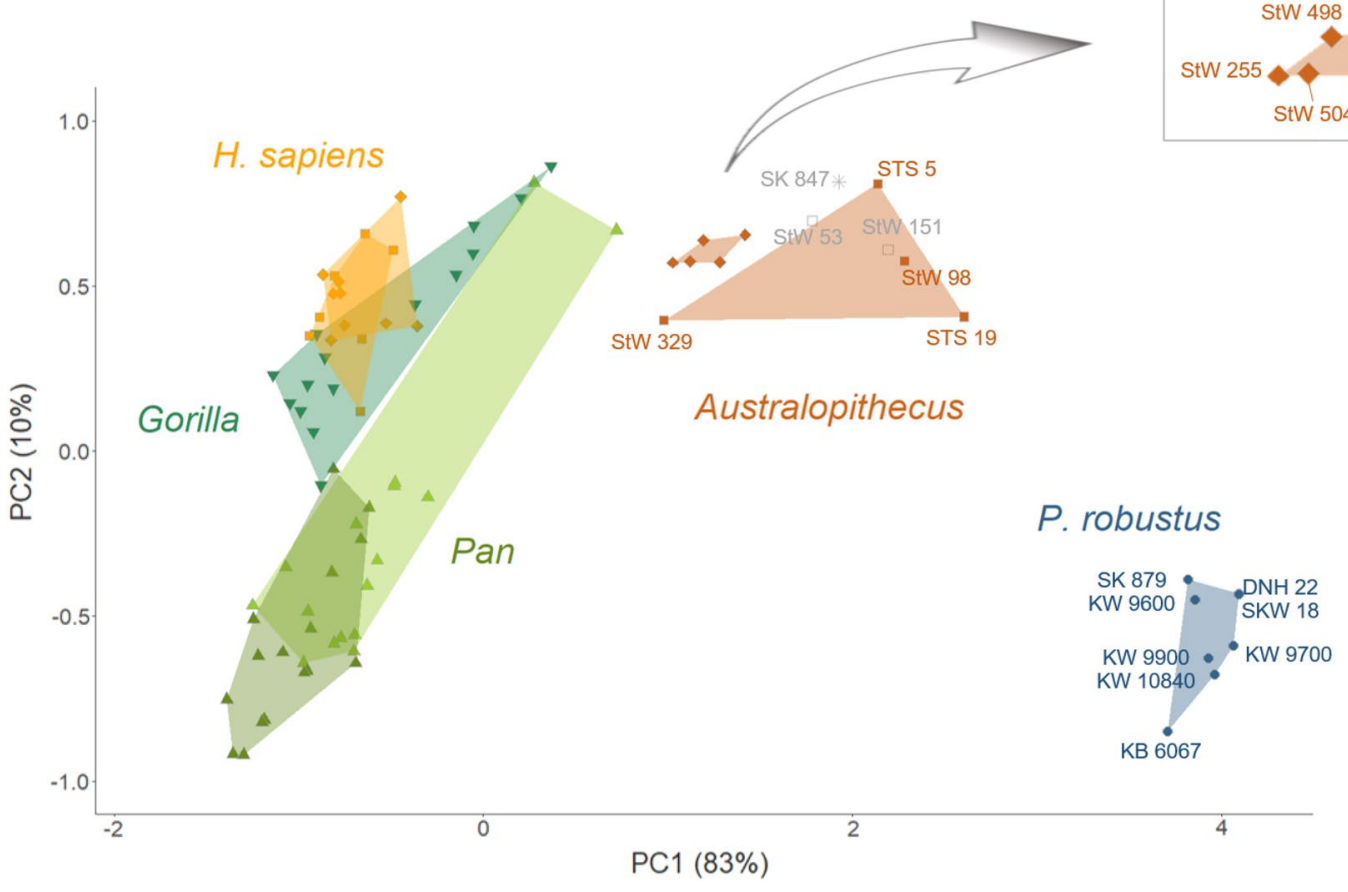

Figure 3. Tangent principal component analysis (TPCA) of cochlear curves compared using curvature and torsion. Blue circles are for $P$. robustus from Kromdraai (including the new specimens from Kromdraai, KW 9600, KW 9700, KW 9900, KW 10840), Swartkrans and Drimolen; brown diamonds and squares are for $A$. africanus from Sterkfontein, including five specimens that have been suggested sample a second species ${ }^{19}$ (diamonds) also illustrated in the enlarged box (top right); light and dark green triangles are for pygmy and common chimpanzees, respectively; green inverted triangles are for gorillas; orange squares and diamonds are for male and female modern humans, respectively; star is for SK 847 (early Homo); open grey squares are for StW 151 and StW 53 (here considered as "indeterminate"). Note that DNH 22 and SKW 18 overlap because their cochlear curves are nearly identical and their PC1 and PC2 values are similar.

affinities of StW 53 and StW 151 (Figs. 3, 4), it should be noted that the cochlear shape of SK 847 is also similar to that of the A. africanus specimen Sts 5 ("Mrs. Ples") as well as to StW 53. When compared with the variation among Pan, Gorilla and Homo sapiens, the different cochlear morphologies of P. robustus and A. africanus are consistent with their generic distinction.

When compared to A. africanus, P. robustus is distinctive in that at any location, from the base to the apex, it shows a lower torsion along its Fréchet mean (Fig. 2). This difference is particularly noticeable towards the cochlea's last turn where the lowest-frequency waves are resolved ${ }^{8,9}$. Near the cochlear apex, the P. robustus Fréchet mean suggests a near absence of torsion and a high degree of curvature, which results in a hook-like shape.

Effects of selection and phylogeny on cochlear shape. To assess whether the morphological differences between $P$. robustus and A. africanus were more likely to be the result of neutral evolution or directional selection $^{27}$, we compared the pattern of maximal between-group variance relative to within-group variance ("Methods") ${ }^{28,29}$. Directional selection assumes a deviation from proportionality between the covariance matrix of related taxa means (B) and the pooled within-taxa covariance matrix (a weighted average of the taxa covariance matrices as an estimate of the ancestral covariance matrix) (W). A principal coordinate analysis of the covariance matrices of TPCA values shows that the within-taxa matrices form a relatively homogeneous cluster around their weighted average $\mathrm{W}$, whereas B falls far outside this cluster (Fig. S3). A significant deviation from proportionality ( $\mathrm{p}$-value $<0.001)$ between $(\mathrm{B})$ and $(\mathrm{W})$ separates $P$. robustus from the other taxa. This result, together with a relative eigenanalysis indicating that the inter-taxa covariance exceeds the variation expected for neutral evolution, is consistent with cochlear shape having evolved under directional selection in P. robustus.

Because the Fréchet mean similarities between more closely-related species are no greater than those between more distantly-related taxa (i.e., there is no (B)/t relationship), variation in cochlear shape did not increase linearly with evolutionary time, as would be expected in the case of a Brownian-motion model of evolution ("Methods") ${ }^{30}$. We therefore used the alternative Ornstein-Uhlenbeck (OU) model that is most suitable for selection $^{30}$, and applied it to the PC1 scores from the TPCA by using two alternative scenarios of rates of cochlear shape change. It is noteworthy that there was a rapid divergence between the A. africanus and P. robustus lineages between 3 and $2 \mathrm{Ma}$, whereas early Homo retained a more ancestral condition than P. robustus (Fig. S4). 


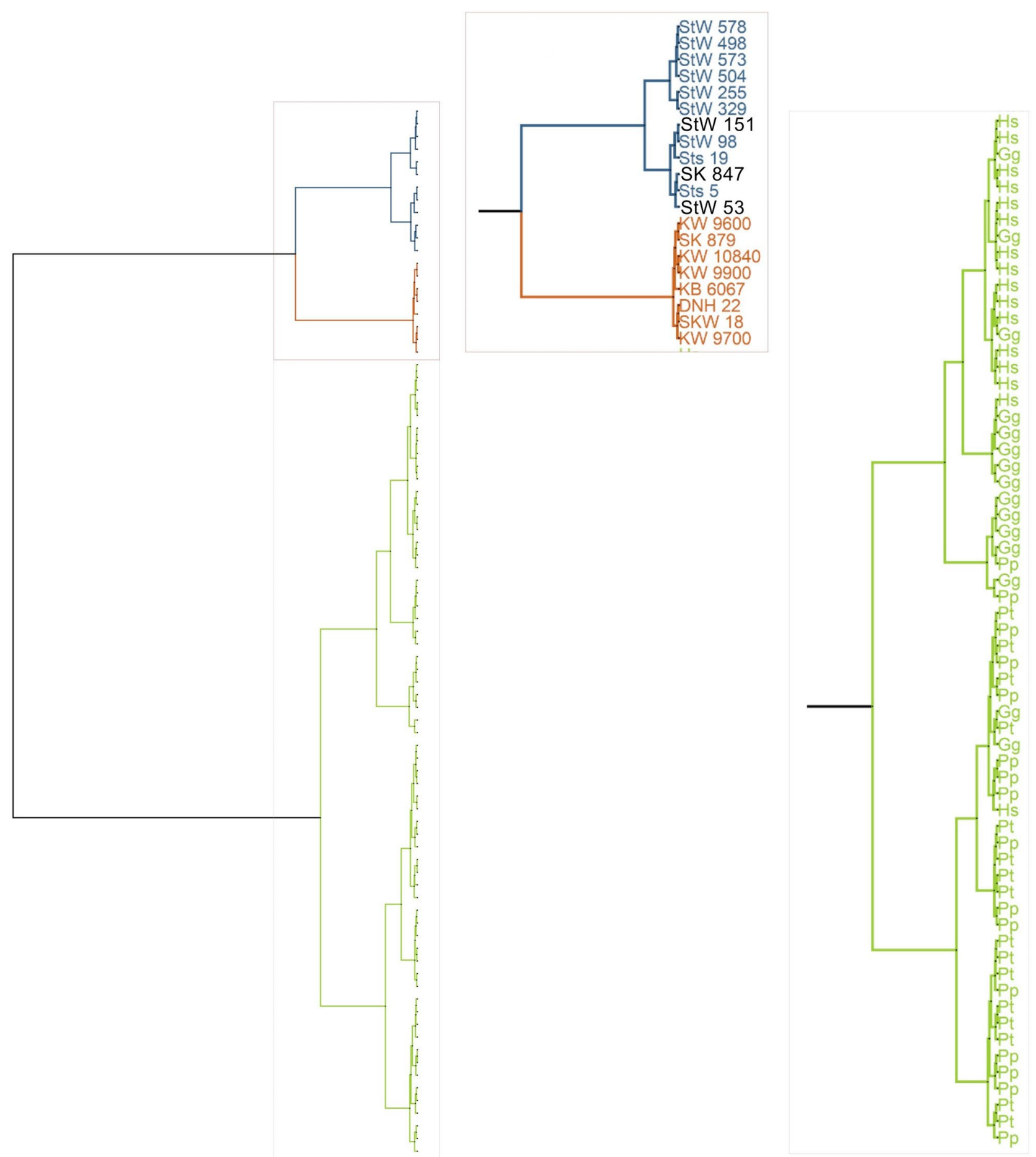

Figure 4. Assessments of the clustering performance of the TPCA. The best classification is achieved with three clusters representing extant species (green color, Hs, Homo sapiens; Gg, Gorilla gorilla; Pp, Pan paniscus; Pt, Pan troglodytes), A. africanus (blue color) and P. robustus (brown color). Note that the SK 847 (early Homo), StW 151 and StW 53 (here considered as 'unknown') are illustrated in black and cluster with A. africanus specimens.

\section{Discussion}

Neither linear measurements nor 3DGM morphometric techniques have been able to discern clear-cut differences between $A$. africanus, $P$. robustus and early Homo with respect to their middle ear ossicles ${ }^{31}$, bony labyrinths $s^{1,2,11}$ or cochleae ${ }^{2,11}$. The present study using diffeomorphometry has shown that the cochlear shapes of both A. africanus and especially P. robustus are distinct from those of modern humans and the African apes. We find genus-level differences between Paranthropus and Australopithecus in southern Africa, but no cogent evidence for a taxonomic distinction within the A. africanus sample from Sterkfontein. In light of the variation 
documented here in A. africanus, morphological diversity is low in P. robustus as sampled at Kromdraai, Swartkrans and Drimolen (Figs. 3, 4). The KNN classification and V-measure from a TPCA of cochlear shape highlights the near similarity between specimens from Swartkrans (SKW 18) and Drimolen (DNH 22) and the absence of morphological change that may be related to time with the $P$. robustus lineage. Thus, evidence from the bony labyrinth is not consistent with microevolution in $P$. robustus ${ }^{21}$, although different results from other regions of the skeleton would perhaps not be surprising.

Mosaic evolution is also evident within the bony labyrinth. For example, with respect to SK 847 and StW 53, while "extreme" differences in their semicircular canal proportions suggest that it is "highly unlikely" they belong to the same early Homo species ${ }^{1}$, their cochlear morphology is remarkably similar.

Recent analyses suggest that Homo and Paranthropus are sister clades, and Bayesian inference has shown high levels of support for A. africanus being the sister taxon to the Homo + Paranthropus clade ${ }^{32}$. Thus, the similarity in cochlear shape between SK 847 (early Homo), StW 53 and StW 151 (here treated as taxonomically indeterminate) and $A$. africanus suggests that the cochlear morphology of southern African early Homo was more conservative compared to P. robustus. Our analyses reveal strong selection for cochlear morphology in P. robustus, and suggest that selection occurred early in the evolutionary history of this species. Stable isotope $\left(\delta^{13} \mathrm{C}\right)$ data and faunal analyses indicate that in southern Africa both P. robustus and early Homo inhabited more open environments than A. africanus ${ }^{33}$. If one posits the co-occurrence of early Homo and P. robustus, the relatively rapid divergence of $P$. robustus cochlear shape from the ancestral condition (Fig. S4) could have resulted in acoustic niche partitioning between these two southern African hominin lineages.

Micromechanical models ${ }^{34}$ consistently suggest that an increased cochlear curvature enhances low-frequency (LF) sensitivity in the apical portion where curvature is the greatest ${ }^{35,36}$. Given our finding of significant size-independent and significant differences in cochlear mean shapes between species, and the unique hook-like shape (i.e., near absence of torsion and high degree of curvature) near the apex in P. robustus, cochlear micromechanics ${ }^{34-36}$ suggest enhanced LF sensitivity in this species. Comparative studies over a range of primates indicate a relationship between LF sensitivity and frequency difference limen (FDL) sensitivity ${ }^{37}$, wherein frequency resolution increases linearly as frequency increases. This enhanced capacity to distinguish between two pure tones would allow individuals to more reliably discriminate species-specific communication signals. Compared to Pan troglodytes, modern humans show a wider auditory band of maximum sensitivity that emphasizes higher frequencies ${ }^{38,39}$ and higher frequency resolution ${ }^{40}$ that would facilitate short-distance calling. In contrast, the cochlear morphology of P. robustus (Fig. 2) suggests an emphasis on higher frequency resolution in the lower frequency range. One possible explanation is that $P$. robustus evolved a novel auditory specific mate recognition system (SMRS). The ability to rapidly discriminate conspecific communication signals is well known in primates ${ }^{40,41}$. Any such auditory SMRS may be influenced by the nature of the habitat in which it is used ${ }^{41}$, and it is possible that higher frequency resolution in the lower frequency range was required for the preferred habitat of $P$. robustus.

We recognize that it is not necessary to interpret all morphological differences in the context of adaptive functionality ${ }^{42}$, but the unique cochlear morphology of $P$. robustus is most likely to have been produced under strong directional selection (Fig. S3). Because the number of specimens (n), the dimensionality of the TPCA space $(\mathrm{p})$, and the ratio $\mathrm{n} / \mathrm{p}$ are important factors for covariance estimation, larger sample sizes would strengthen the statistical power of this analysis. Our results nevertheless suggest that the distinctive cochlear shape of $P$. robustus represents a unique auditory adaptation among southern African hominins. The phylogenetic results also lend support to the hypothesis that there was rapid, directional evolution of cochlear shape in P. robustus. The obvious question is whether this cochlear shape is autapomorphic for P. robustus, or whether it is shared by its congeners in eastern Africa. If it is, this would be compelling evidence for Paranthropus monophyly, although if it is not, this would not constitute evidence against monophyly. Of equal interest is whether the eastern African representatives of early Homo exhibit the same configuration as their congeners from southern Africa. Cochlear morphology has the potential to improve our understanding of the paleobiology of extinct hominin taxa.

\section{Methods}

Taxonomic attribution of the new fossil hominin specimens from Kromdraai. Among the four new fossil hominin specimens from Kromdraai (Text S1, Table S2, Fig. S1) one is an adult (KW 9900) and three are juveniles (KW 9600, KW 9700, KW 10840). The juvenile status was assessed by measuring the degree of opening of the subarcuate fossa ${ }^{26,44,45}$, a canal that extends through the arc of the anterior semi-circular canal and gradually obliterates during development. The small variation in the degree of fossa closure between KW 9600 (7.2\%, mean of right and left sides), KW 9700 (6.4\%, left side) and KW 10,840 (5.8\%, mean of right and left sides) suggest that they represent three close-in-age juvenile individuals. Three other $P$. robustus specimens in our sample are also juveniles: (i) KB 6067 represents the youngest specimen as judged from the degree of its fossa closure ${ }^{26}$, (ii) SKW 18 is clearly associated with SK 52 to form a compound skull ${ }^{46}$, and (iii) TM $1517^{43}$. The two latter specimens show unerupted M3s with some root development internally visible from their micro-CTs (pers. obs). Three Australopithecus specimens also represent juvenile individuals. The higher degree of opening of the fossa in StW 98 (17.8\%) suggest it is younger than StW 329 (5.8\%) and StW 255 (4.5\%). The juvenile status of StW 151 is confirmed by its opened fossa (5.2\%). We investigated whether KW 9600, KW 9700, KW 9900 and KW 10840 showed more similarities to P. robustus from Kromdraai (TM 1517 and KB 6067), Swartkrans (SK 879 and SKW 18) and Drimolen (DNH 22), or to early Homo (SK 847). To do so, the micro-CT data were resliced in a plane that best fitted the horizontal semi-circular canal. One of us (JB) measured the transverse labyrinthine index (TLI), the inclination of the ampular line and the cochlear basal turn relative to the orientation of the horizontal (or lateral) semi-circular canal (APA $<\mathrm{LSCm}$ and COs $<\mathrm{LSCm}$, respectively $)^{47}$. Six additional indices of the bony labyrinth were added: the arc lengths of the horizontal (HZ), posterior (PO) and anterior (AN) 
semi-circular canals (noted HZL, POL and ANL, respectively) measured by placing a curve along their outer circumference. We then computed the HZL/ANL, HZL/POL and POL/ANL indices. The length of the cochlea $\left(\mathrm{ECL}^{26}\right)$ was also size-standardized by HZL, POL and ANL and provided three additional indices (ECL/HZL, $\mathrm{ECL} / \mathrm{POL}$ and ECL/ANL). We ensured the best-possible measurements (taken with the Avizo software package, www.vsg3d.com/avizo) by using simultaneously the resliced stacks of micro-CT images and the $3 \mathrm{D}$ reconstruction of the bony labyrinths. In order to assess potential measurement errors, each variable was measured twice on five randomly selected bony labyrinths with more than a one-day interval between each trial. The intraclass correlation coefficient results for the reproducibility of the measurements showed good agreement (ICC $>0.8)$. In order to assess the compatibility between our measurements of APA $<$ LSCm, COs $<$ LSCm and TLI and the ones made on the same specimens from medical $\mathrm{CTs}^{47}$, we compared the two sets of measurements for TM 1517, SK 879 and SK 847 (Table S3). We observed identical or very similar values. The bony labyrinth of the type specimen of $P$. robustus from Kromdraai (TM 1517) ${ }^{43}$ can be measured only partly (Text S2) due to precipitated crystallized material within the bone that prevents the accurate measure of cochlear shape.

Ethical approval. All the steps of the present study were performed in accordance with relevant guidelines and regulations. No data used in this study involved experimentation, risk or constraint added by the research. Only museum specimens were included in this study. We obtained permissions to access these museum specimens that have already been used in several published studies ${ }^{2,15,26}$.

Cochlear shape: maps of curvature and torsion. The use of 3DGM assumes that cochlear curves are linear (or Euclidian) spaces, whereas they are not. Non-linear methods are needed to analyze cochlear pure shape ${ }^{12,13}$ in 3D. Therefore, we used diffeomorphometry, a method based on the uniform scaling and nonlinear (elastic) registration of 3D non-linear spaces (called "Riemannian manifolds") ${ }^{12,13}$ that has been applied in evolutionary anthropology ${ }^{48,49}$. Its efficacy for taxonomic discrimination of fossil hominin teeth has been compared to results obtained with $3 \mathrm{DGM}^{14}$. The $3 \mathrm{DGM}$ cannot capture key geometrical properties of $3 \mathrm{D}$ cochlear curves, with their two most important properties: the curvature (bending) and the torsion (twisting) that can be defined intuitively as follows. Curvature describes the degree of bending at any location along the cochlear curve from the base to the apex. The greater the curvature, the faster is the change of its tangent vector direction. Torsion describes how the 3D curve twists out the plane of curvature. The curvature and torsion were calculated (with Matlab 2016a) by using the Frenet-Serret formulae ${ }^{50}$ that can be described intuitively as follows. A curve can be regarded as the path of a moving particle. We measure its bending by using the curvature as the rate of change for the unit tangent vector (normalized first derivative). We also measure how quickly the curve twists by using the rate of change of the oscillating plane by the tangent vector and the normal vector. We emphasize here that our mapping of the changes in curvature along the $3 \mathrm{D}$ cochlear curve (Fig. 2) is fundamentally different from the global assessment of curvature represented by only one measure such as the "radii ratio"2,9, which is the ratio of the radii of the basal and apical termini of the cochlear curve. Assessments of "radii ratio" obtained from photographs ${ }^{51}$ embed the cochlear curve within a plane and, therefore, cannot capture 3D shapes and torsion and cannot be compared with our measurements. Moreover, since "radii ratio" overlap between P. robustus and A. africanus, as well as between species with larger (e.g., gorillas) and smaller (e.g., pygmy chimpanzees) skull bases $^{2}$, the global curvature of the cochlea is not higher when this organ is "packed" in smaller cranial bases.

Cochlear curve: sampling and analysis. After the segmentation of each bony labyrinth from its microCT volumes, we placed the cochlear curve as already described ${ }^{2}$ and we resampled it to 200 semi-landmarks. In order to assess potential errors in the placement of the curves, we selected randomly ten cochleae. One of us (JB) placed the curves twice on each cochlea with more than a one-week interval between each session. The significance of Procrustes distances within and between the two repeated measurements was assessed via permutation tests (1000 random permutations). They indicated that the differences in shape between specimens were significantly higher $($ at $\mathrm{p}<0.01)$ than those within repeated measurements. To compute elastic deformations between two corresponding cochlear curves, we adapted the general shape analysis framework ${ }^{16}$. These deformations are generated using vector fields between curves under an elastic Riemannian metric (Text S3). The computations on Riemannian manifolds and the TPCA were performed in Matlab 2016a by using the packages 'SRVF_FDA'16 and 'ROPTLIB' 52 . The TPCA can also be performed by using the R package 'shapes' ${ }^{53}$.

Assessment of the clustering performance of the TPCA results. We performed K-nearest neighbour (KNN) classification (implemented in the R package 'yardstick ${ }^{54}$ ) in which each specimen in the sample is assigned a class (here, a taxon) depending on its similarities with its neighbours in TPCA space (Text S4). This method considers imbalanced class sizes to account for underrepresentation of small samples classes. The performance of this classification is defined as the average of the true positive rates of each class. To evaluate the clustering, we use the $\mathrm{V}$-measure ${ }^{55}$ (implemented in the $\mathrm{R}$ package 'sabre ${ }^{56}$ ), a metric that is independent of the size of the data, the number of classes, and the number of clusters (i.e., the clustering).

Analysis of Procrustes coordinates of landmark data. We performed a generalized Procrustes superimposition of the landmark coordinates and a PCA with the R packages 'Morpho v2.8'57 and 'geomorph v3.3.2 ${ }^{58}$ ). We did not use between-groups PCA (bgPCA) because of the problems that have been noted when using fewer groups than variables ${ }^{59}$. Moreover, because of fundamental differences between 3DGM and our approach based on diffeomorphisms (see above), we expect that bgPCA applied on Procrustes coordinates will not improve the delineation of fossil taxa. 
Comparison of variance-covariance patterns of differences within and between taxa. Relative eigenanalysis ${ }^{28}$ was done with the 'vcvComp' R package ${ }^{29}$. We used the three first PCs of the TPCA results $(83 \%$, $10 \%$ and $4 \%$ of variance represented by PC1, PV2 and PC3, respectively) and six taxa to perform this analysis. Indeed, when we evaluated the clustering performance of our TPCA, we concluded that all the Australopithecus specimens in our sample were more likely grouped in one species: A. africanus. The maximum likelihood (ML) test suggested a deviation from proportionality between $B$ and $W(p=0.00106)$ and thus the action of selective forces. Since this test does not specify the magnitude of deviation from proportionality, we performed an ordination of the covariance matrices, together with $\mathrm{W}$ and $\mathrm{B}$. Relative to the heterogeneity of taxa covariance matrices, B clearly deviates from W along the first principal coordinate (Fig. S3) and we reject the null hypothesis that the matrices are proportional to each other. The covariance matrices of gorillas, chimpanzees and modern humans group close together, in close proximity to W. The covariance matrices of $P$. robustus, A. africanus and specimens attributed to early Homo deviate from this pattern, with P. robustus being an outlier. A relative PCA of B with respect to $\mathrm{W}$ provides the following three successive eigenvalues: $44.79,5.89$ and 0.24 . The first relative eigenvalue is more than 7 times larger than the second one, which is significant at $p<0.001$, and similarly for the last relative eigenvalue. The first and second relative eigenvalues (44.79 and 5.89) largely exceed the threshold estimated on the basis of genetic data by using the $F_{S T}$ test $^{60}$. $F_{S T}$ represents the fraction of genetic diversity attributable to between-group differences. Under pure genetic drift, $F_{S T}$ values among extant humans and African apes range between 0.09 to 0.42 within species, and between 0.49 to 0.94 among species ${ }^{61,62}$.

Ornstein-Uhlenbeck (OU) process. Since we did not find a relationship between (B) and evolutionary time since divergence ( $\mathrm{t}$ ) (as expected in Brownian motion), we fitted a OU process to the TPCA scores on PC1. We used a phylogeny that combines a calibration with phylogenomics ${ }^{63}$ and fossil evidence fixing the ages of some internal branches, nodes and tips ${ }^{64}$. Because the phylogenetic position of A. africanus and P. robustus is not resolved, we simulated the changes of TPCA scores on PC1 along each of two alternative scenarios (Fig. S4): (i) an evolutionary continuity between $A$. africanus and P. robustus, the latter being considered as a sister clade of Homo with a MRCA set at $2.8 \mathrm{Ma}$; (ii) P. robustus and Homo with a MRCA set at $3.5 \mathrm{Ma}$ and with A. africanus considered as a sister group to both. The optimal hyper-parameter was estimated with Markov-Chain Monte Carlo (MCMC) simulations from a maximum total of 5.000.000 iterations.

\section{Data availability}

The datasets generated during and/or analyzed during the current study are available from the corresponding author on request. Some datasets and codes supporting the current study have not yet been deposited in a public repository because they are part of further investigation.

Received: 17 March 2021; Accepted: 11 August 2021

Published online: 23 August 2021

\section{References}

1. Spoor, F., Wood, B. A. \& Zonneveld, F. Implications of early hominid labyrinthine morphology for evolution of human bipedal locomotion. Nature 369, 645-648 (1994).

2. Braga, J. et al. Disproportionate cochlear length in genus Homo shows a high phylogenetic signal during apes' hearing evolution. PLOS ONE 10, e0127780 (2015).

3. Urciuoli, A. et al. The evolution of the vestibular apparatus in apes and humans. Elife 9, e51261 (2020).

4. Ward, D. L. et al. Early life malnutrition and fluctuating asymmetry in the rat bony labyrinth. Anat. Rec. https://doi.org/10.1002/ ar.24601 (2021).

5. Gunz, P., Ramsier, M., Kuhrig, M., Hublin, J.-J. \& Spoor, F. The mammalian bony labyrinth reconsidered, introducing a comprehensive geometric morphometric approach. J. Anat. 220, 529-543 (2012).

6. Grohé, C., Tseng, Z. J., Lebrun, R., Boistel, R. \& Flynn, J. J. Bony labyrinth shape variation in extant Carnivora: A case study of Musteloidea. J. Anat. 228, 366-383 (2016).

7. Mennecart, B. et al. Bony labyrinth morphology clarifies the origin and evolution of deer. Sci. Rep. 7, 13176 (2017).

8. Vater, M. \& Kössl, M. Comparative aspects of cochlear functional organization in mammals. Hear. Res. 273, 89-99 (2011).

9. Manoussaki, D. et al. The influence of cochlear shape on low-frequency hearing. Proc. Natl. Acad. Sci. U.S.A. 105, 6162-6166 (2008).

10. Wannaprasert, T. \& Jeffery, N. Variations of mammalian cochlear shape in relation to hearing frequency and skull size. Trop. Nat. Hist. 15, 41-54 (2015).

11. Beaudet, A. The inner ear of the Paranthropus specimen DNH 22 from Drimolen, South Africa. Am. J. Phys. Anthropol. 170, $439-446$ (2019).

12. Kendall, D. G. Shape manifolds, procrustean metrics and complex projective spaces. Bull. Lond. Math. Soc. 16, 81-121 (1984).

13. Srivastava, A. \& Klassen, E. Functional and Shape Data Analysis (Springer, 2016).

14. Braga, J. et al. Efficacy of diffeomorphic surface matching and 3D geometric morphometrics for taxonomic discrimination of Early Pleistocene hominin mandibular molars. J. Hum. Evol. 130, 21-35 (2019).

15. Braga, J. et al. Cochlear shape reveals that the human organ of hearing is sex-typed from birth. Sci. Rep. 9, 10889 (2019).

16. Srivastava, A., Klassen, E., Joshi, S. H. \& Jermyn, I. H. Shape analysis of elastic curves in Euclidean spaces. IEEE Trans. Pattern Anal. Mach. Intell. 33, 1415-1428 (2011).

17. Grine, F. E. The alpha taxonomy of Australopithecus africanus. In The Paleobiology of Australopithecus (eds Reed, K. E. et al.) 73-104 (Springer, 2013).

18. Grine, F. E., Delanty, M. M. \& Wood, B. A. Variation in mandibular postcanine dental morphology and hominin species representation in Member 4, Sterkfontein, South Africa. In The Paleobiology of Australopithecus (eds Reed, K. E. et al.) 125-146 (Springer, 2013).

19. Clarke, R. J. Australopithecus from Sterkfontein caves, South Africa. In The Paleobiology of Australopithecus (eds Reed, K. E. et al.) 105-123 (Springer, 2013).

20. Wood, B. A. \& Boyle, E. K. Hominin taxic diversity: Fact or fantasy?. Yearb. Phys. Anthropol. 159, S37-S78 (2016). 
21. Martin, J. M. et al. Drimolen cranium DNH 155 documents microevolution in an early hominin species. Nat. Ecol. Evol. 5, 38-45 (2020).

22. Rak, Y., Kimbel, W. H., Moggi-Cecchi, J., Lockwood, C. A. \& Menter, C. The DNH 7 skull of Australopithecus robustus from Drimolen (Main Quarry). South Africa. J. Hum. Evol. 151, 102913 (2021).

23. Moggi-Cecchi, J., Tobias, P. V. \& Beynon, A. D. The mixed dentition and associated skull fragments of a juvenile fossil hominid from Sterkfontein South Africa. Am. J. Phys. Anthropol. 106, 425-465 (1998).

24. Curnoe, D. \& Tobias, P. V. Description, new reconstruction, comparative anatomy, and classification of the Sterkfontein Stw 53 cranium, with discussions about the taxonomy of other southern African early Homo remains. J. Hum. Evol. 50, 36-77 (2006).

25. Clarke, R. J. Latest information on Sterkfontein's Australopithecus skeleton and a new look at Australopithecus. S. Afr. J. Sci. 104, 443-449 (2008).

26. Braga, J. et al. A new partial temporal bone of a juvenile hominin from the site of Kromdraai B (South Africa). J. Hum. Evol. 65, $447-456$ (2013).

27. Lande, R. Natural selection and random genetic drift in phenotypic evolution. Evol. 30, 314-334 (1976).

28. Bookstein, F. \& Mitteroecker, P. Comparing covariance matrices by relative eigenanalysis, with applications to organismal biology. Evol. Biol. 41, 336-350 (2014).

29. Le Maitre, A. \& Mitteroecker, P. Multivariate comparison of variance in R. Methods Ecol. Evol. 10, 1380-1392 (2019).

30. Beaulieu, J. M., Jhwueng, D. C., Boettiger, C. \& O’Meara, B. C. Modeling stabilizing selection: expanding the Ornstein-Uhlenbeck model of adaptive evolution. Evol. 66, 2369-2383 (2012).

31. Quam, R. et al. Early hominin auditory ossicles from South Africa. Proc. Natl. Acad. Sci. U.S.A. 110, 8847-8851 (2013).

32. Mongle, C. S., Strait, D. S. \& Grine, F. E. Expanded character sampling underscores phylogenetic stability of Ardipithecus ramidus as a basal hominin. J. Hum. Evol. 131, 28-39 (2020).

33. Sponheimer, M. \& Lee-Thorp, J. A. Biogeochemical evidence for the environments of early Homo in South Africa. In The First Humans: Origin and Early Evolution of the Genus Homo (eds Grine, F. E. et al.) 185-194 (Springer, 2009).

34. Ni, G., Elliott, S. J., Ayat, M. \& Teal, P. D. Modelling cochlear mechanics. Biomed. Res. Int. 2, 150637 (2014).

35. Cai, H., Manoussaki, D. \& Chadwick, R. Effects of coiling on the micromechanics of the mammalian cochlea. J. R. Soc. Interface 2, 341-348 (2005).

36. Tang, Z. et al. Research on the characteristics of dynamic behavior of basilar membrane in spiral cochlea. J. Vibroengineering 19, 3809-3821 (2017).

37. Osmanski, M. S., Song, X., Guo, Y. \& Wang, X. Frequency discrimination in the common marmoset (Callithrix jacchus). Hear. Res. 341, 1-8 (2016).

38. Quam, R. M. et al. Early hominin auditory capacities. Sci. Adv. 1, e1500355 (2015).

39. Kojima, S. Comparison of auditory functions in the chimpanzee and human. Folia Primatol. 55, 62-72 (1990).

40. Machens, C. K. et al. Single auditory neurons rapidly discriminate conspecific communication signals. Nat. Neurosci. 6, 341-342 (2003).

41. Zoloth, S. R. et al. Species-specific perceptual processing of vocal sounds by monkeys. Science 204, 870-873 (1979).

42. Gould, S. J. \& Lewontin, R. C. The spandrels of San Marco and the Panglossian paradigm: A critique of the adaptationist programme. Proc. R. Soc. Lond. B. 205, 581-598 (1979).

43. Broom, R. The Pleistocene anthropoid apes of South Africa. Nature 142, 377-379 (1938).

44. Coqueugniot, H. et al. Early brain growth in Homo erectus and implications for cognitive ability. Nature 431, 299-302 (2004).

45. Carayon, D., Vaysse, F., Tramini, P., Dumoncel, J. \& Esclassan, R. The age-related maturational pattern of the human subarcuate fossa. C. R. Palevol. 14, 139-145 (2015).

46. Clarke, R.J. The cranium of the Swartkrans hominid SK 847 and its relevance to human origins. Ph.D. Thesis (University of the Witwatersrand,1977).

47. Spoor, F. The comparative morphology and phylogeny of the human bony labyrinth. Ph.D. Thesis. (Utrecht University, 1993).

48. Boyer, D. M. et al. Algorithms to automatically quantify the geometric similarity of anatomical surface. Proc. Natl. Acad. Sci. U.S.A. 108, 18221-18226 (2011).

49. Durrleman, S., Pennec, X., Trouvé, A., Ayache, N. \& Braga, J. Comparison of the endocranial ontogenies between chimpanzees and bonobos via temporal regression and spatiotemporal registration. J. Hum. Evol. 62, 74-88 (2012).

50. Kim, K., Kim, P. T., Koo, J. \& Pierrynowski, M. R. Frenet-Serret and the estimation of curvature and torsion. IEEE J. Select. Top. Sig. Process. 7, 646-654 (2013).

51. Pietsch, M. et al. Spiral form of the human cochlea results from spatial constraints. Sci. Rep. 7, 7500 (2017).

52. Huang, W., Absil, P.-A., Gallivan, K. \& Hand, P. ROPTLIB: an object-oriented C++ library for optimization on Riemannian manifolds (2016). https://www.math.fsu.edu/ whuang2/Indices/index_ROPTLIB.html

53. Dryden, I.L. \& Mardia, K.V. Statistical Shape analysis, with Applications in R. Second Edition (John Wiley and Sons, 2016). R package version 1.2.6. https://cran.r-project.org/web/packages/shapes/index.html

54. Kuhn, M. \& Vaughan, D. Package 'yardstick'. Tidy Characterizations of Model Performance. R package version 0.0.7. https:// CRAN.R-project.org/package=yardstick (2020).

55. Rosenberg, A. \& Hirschberg, J. V-Measure: A Conditional Entropy-Based External Cluster Evaluation Measure. Joint Conference on Empirical Methods in Natural Language Processing and Computational Natural Language Learning, 410-420 (2007).

56. Nowosad, J. \& Stepinski., T.F. ,. Spatial association between regionalizations using the information-theoretical V-measure. Int. J. Geogr. Inf. Sci. 32, 1-16 (2018).

57. Schlager, S. \& Morpho, R. Shape analysis in R. In Statistical Shape and Deformation Analysis (eds Zheng, G. et al.) 217-256 (Academic Press, 2017).

58. Adams, D., Collyer, M., Kaliontzopoulou, A. \& Baken, E. Geomorph: Software for geometric morphometric analyses. R package version 3.3.2. https://cran.r-project.org/package=geomorph (2021).

59. Cardini, A., O’Higgins, P. \& Rohlf, F. J. Seeing distinct groups where there are none: Spurious patterns from between group PCA. Evol. Biol. 46, 303-316 (2019).

60. Holsinger, K. E. \& Weir, B. S. Genetics in geographically structured populations: Defining, estimating and interpreting FST. Nat. Rev. Genet. 10, 639-650 (2009).

61. Fischer, A., Pollack, J., Thalmann, O., Nickel, B. \& Pääbo, S. Demographic history and genetic differentiation in apes. Curr. Biol. 16, 1133-1138 (2006).

62. Fischer, A. et al. Bonobos fall within the genomic variation of chimpanzees. PLoS ONE 6, e21605 (2011).

63. Dos Reis, M. et al. Using phylogenomic data to explore the effects of relaxed clocks and calibration strategies on divergence time estimation: primates as a test case. Syst. Biol. 67, 594-615 (2018).

64. Rieux, A. \& Balloux, F. Inferences from tip-calibrated phylogenies: A review and a practical guide. Mol. Ecol. 25, 1911-1924 (2016).

\section{Acknowledgements}

We thank Rolf Quam for informative discussions and comments on an earlier version of this manuscript. We thank anonymous reviewers whose comments helped to improve the manuscript. We thank the South African Heritage Resources Agency (SAHRA), the Evolutionary Studies Institute, University of the Witwatersrand, for 
curating fossils from Kromdraai and the Ditsong National Museum of Natural History for giving us access to the fossils under their care. We also thank Sifelani Jirah for access to specimens and Frikkie de Beer, Jakobus Hoffman for scanning.

\section{Author contributions}

J.B. and C.S. designed the research; J.B., J.F.T., B.Z. and K.J. collected the data; J.B., C.S., A.F., Y.F., V.Z. and M.M. conducted the analyses; J.B., F.G. and B.W. wrote the paper with contributions from C.S. and V.Z. All the authors edited the text.

\section{Funding}

This work was supported by the French Ministry of Foreign Affairs (Commission des fouilles), the PhyloFormes3D project from the Centre National de la Recherche Scientifique (CNRS), the Institut des Déserts et des Steppes (Paris), the "Patrick Mathieu Recherche et Conseil" company, the Institut Picot de Lapeyrouse (Toulouse) and the "AESOP plus" programme of Erasmus Mundus (European Union).

\section{Competing interests}

The authors declare no competing interests.

\section{Additional information}

Supplementary Information The online version contains supplementary material available at https://doi.org/ 10.1038/s41598-021-96543-w.

Correspondence and requests for materials should be addressed to J.B.

Reprints and permissions information is available at www.nature.com/reprints.

Publisher's note Springer Nature remains neutral with regard to jurisdictional claims in published maps and institutional affiliations.

(c) (i) Open Access This article is licensed under a Creative Commons Attribution 4.0 International License, which permits use, sharing, adaptation, distribution and reproduction in any medium or format, as long as you give appropriate credit to the original author(s) and the source, provide a link to the Creative Commons licence, and indicate if changes were made. The images or other third party material in this article are included in the article's Creative Commons licence, unless indicated otherwise in a credit line to the material. If material is not included in the article's Creative Commons licence and your intended use is not permitted by statutory regulation or exceeds the permitted use, you will need to obtain permission directly from the copyright holder. To view a copy of this licence, visit http://creativecommons.org/licenses/by/4.0/.

(C) The Author(s) 2021 